\title{
Comparison of the 2- and 6-Minute Walk Test in multiple sclerosis
}

Gijbels $\mathrm{D}^{1,2 \S}$, Eijnde $\mathrm{BO}^{1,2^{*}}$, Feys $\mathrm{P}^{1,2,3^{*}}$

${ }^{1}$ REVAL Rehabilitation Research Center, Hasselt University, Agoralaan Building A, BE3590 Diepenbeek, Belgium

${ }^{2}$ BIOMED Biomedical Research Institute, Hasselt University, Agoralaan Building A, BE3590 Diepenbeek, Belgium

${ }^{3}$ Katholieke Universiteit Leuven, Departments of Biomedical Kinesiology and Rehabilitation Sciences, Tervuursevest 101, BE-3001 Leuven, Belgium

${ }^{\S}$ Corresponding author: $\quad(\mathrm{t})+32(0) 478 / 555225$;

(e) domien.gijbels@uhasselt.be;

$$
\text { (f) }+32(0) 11 / 294953
$$

*These authors contributed equally to this work

Keywords: 6-Minute Walk Test, 2-Minute Walk Test, multiple sclerosis

Disclosure: The authors report no conflicts of interest 


\section{Short summary}

The 6-Minute Walk Test (6MWT) is often applied to assess walking distance in multiple sclerosis (MS) but can be both time consuming for the investigator and exhausting for the person with MS (pwMS). In present report, we compared the 6MWT scores of 40 ambulatory pwMS with their scores on the shorter 2-Minute Walk Test (2MWT). The 2MWT enabled to estimate the 6MWT result with a mean relative error of $5 \%\left(R^{2}=0.96 ; p<0.01\right)$. Also, the last 4-minute period of the 6MWT seems redundant. Consequently, the 2MWT may be considered as a practical replacement for the $6 \mathrm{MWT}$ in routine clinical assessment. 


\section{Introduction}

Examination of functional mobility in clinical practice frequently includes an assessment of the distance a person can walk during a given time period. The 6-Minute Walk Test (6MWT) is mostly used to measure walking distance across different study populations,(1-3) with normative data available for comparison.(1) In multiple sclerosis (MS), the 6MWT was found to be a feasible, reliable and responsive measure. $(4,5)$ Although test results were shown to correlate strongly with community ambulation,(6) clinicians may be reluctant to implement this walking capacity test. The time it takes for the 6MWT and the burden to MS subjects, especially in those with increased disability, are important concerns.

The 2-Minute Walk Test (2MWT) may be a pragmatic alternative for the 'gold standard' 6MWT. This shorter test has been validated in subjects with respiratory disease and stroke, $(7,8)$ as well as sporadically being applied as an outcome measure in MS rehabilitation trials.(9) However, it is still unclear if the 2MWT and 6MWT provide similar clinical information in persons with MS (pwMS).

The objective of this study was to compare performances on the 2MWT and 6MWT in a sample of ambulatory pwMS. It was hypothesized that both measures capture the same aspects of mobility. 


\section{Methods}

A convenience sample was recruited among pwMS scheduled at the REVAL Rehabilitation Research Center and the Rehabilitation \& MS Center Overpelt, Belgium. Included subjects ( $n=40)$ had a definite diagnosis of MS according to the McDonald criteria and preservation of at least some ambulatory function (Expanded Disability Status Scale (EDSS) 1.5-6.5, mean score $3.4 \pm 1.2$; age $48 \pm 10$ years; disease duration $11 \pm 7$ years), with 8 of them needing a walking aid (i.e. a cane, crutch(es) or rollator). Subjects had not experienced an exacerbation in the month prior to testing, and had no other medical conditions that interfered with walking. All subjects consented to participate in a cross-sectional research protocol that was approved by the appropriate ethical committees.

Administration of the 2MWT and 6MWT occurred in random order, as decided by a pre-ordered sealed envelope, and was separated by a 30-minute rest period. Walk tests were administered by the same investigator, adopting the instruction script of Goldman et al.(4) Subjects walked, at maximal effort, back and forth in a 30-meter hallway turning round cones, and were permitted to use their habitual assistive devices. Distances walked per minute and total distance were registered. Subjects also completed the 12-item MS Walking Scale (MSWS-12; range 0-100) and the 9-item physical subindex of the Modified Fatigue Impact Scale (MFIS; range 0-36), respectively questioning self-perceived walking ability and physical fatigue.

Data were checked for normality through means of the Shapiro-Wilk statistic. A repeated measures analysis of variance was applied to compare the walking distances under the $2 \mathrm{MWT}$ and the first 2 minutes of the $6 \mathrm{MWT}\left(2^{\prime}, 6 \mathrm{MWT}\right)$. An one-way analysis of variance examined whether the subjects' walking pace differed during minute intervals of the 6MWT. An univariate regression analysis was used to assess the association between the 2MWT and the 6MWT. The obtained regression equation was implemented to plot the 
estimated outcome of the 6MWT versus the actual 6MWT result, this in terms of absolute error as well as relative error (i.e. (100*absolute error)/actual 6MWT result). Additionally, Spearman Rank correlations were computed to appraise the relationship between the walk tests and the EDSS, MSWS-12 and MFIS physical subindex. All analyses were performed using Statistica (Statsoft Inc, Tulsa, USA), with level of significance set at $p<0.05$.

\section{Results}


All data were distributed normally. Total distances walked under the 2MWT and 6MWT were $149 \pm 48$ (range $30-223)$ and $421 \pm 145 \mathrm{~m}(70-666)$ respectively.

Walking distances across both 1-minute intervals of the 2MWT and 2' $6 \mathrm{MWT}$ did not differ significantly ( $p=0.82$ ). Both 2MWT and 2' 6 MWT showed the same, albeit nonsignificant decrease in mean distance over time (i.e. 3 m; see Figure 1). Walking distances across 1-minute intervals of the 6MWT, did not differ significantly $(p=0.94)$ and wereconstant during the last 4-minute period while highly correlated ( Pearson correlation coefficients, $r=0.99 ;$ see also Figure 1).

The 2MWT explained the 6MWT's variation to a very high degree $\left(R^{2}=0.96 ; p<0.01\right)$. The obtained regression equation $(6 \mathrm{MWT}=2,95 * 2 \mathrm{MWT}-19,19)$ enabled to estimate the actual 6MWT result with a mean absolute error of $21 \pm 12 \mathrm{~m}(1-44)$, corresponding to a relative error of $5 \pm 4 \%(0-10)$; this is illustrated in Figure 2 .

On average, subjects rated themselves $53 \pm 21$ (19-90) on the MSWS-12 and 20 46 (1130) on the MFIS physical subindex. The 2MWT and 6MWT produced, respectively, equivalent Spearman Rank correlations with the EDSS ( $r=-0.61 ; r=-0.65)$, MSWS-12 ( $r=-$ $0.72 ; \mathrm{r}=-0.67)$, and MFIS physical subindex $(\mathrm{r}=-0.31 ; \mathrm{r}=-0.29)$.

\section{Discussion}


This report is one of few studies that validates the use of the shorter 2MWT as a measure of walking distance. $(7,8)$ The strong association between the $2 \mathrm{MWT}$ and $6 \mathrm{MWT}$ provides evidence that these two walk tests capture the same aspects of mobility in ambulatory pwMS.

Based on individual 2MWT scores, true performance on the 6MWT could be well estimated in all subjects. Largest absolute errors were seen in pwMS with mild ambulatory dysfunction (cf. Figure 2), but still, relatively, estimation errors did never exceed 10\%. Contrasted with the 6MWT's minimal detectable change and minimally important change of respectively 92 and $55 \mathrm{~m}$ in pwMS (EDSS 0-6.5),(5) the current mean absolute error of $21 \mathrm{~m}$ may be regarded as fairly low.

Besides, in accordance with the observations of Butland et al. (1982),(7) subjects paced themselves, after an initial 'burst' during the 2' $6 \mathrm{MWT}$, constantly during the last 4 minutes of the 6MWT. Also, the $2 \mathrm{MWT}$ and $6 \mathrm{MWT}$ related equally to overall disability and physical fatigue, while we previously found them both to be good estimators for community ambulation.(6) In respiratory disease, the two walk test already demonstrated analogous responsiveness.(10)

In sum, it seems justified to considerthe $2 \mathrm{MWT}$ as practical replacement for the 6MWT in routine clinical assessment (cf. time, burden, and life-long management of pwMS). Follow-up research is now warranted to determine and directly compare the 2MWT and 6MWT's sensitivity to change subsequent to intervention.

\section{Acknowledgements}

DG is recipient of a PhD Fellowship from the Research Foundation Flanders (FWO). The FWO is acknowledged for their Research Grant to PF, the Belgian Charcot Foundation for 
their Equipment Grant. The Rehabilitation \& MS Center Overpelt is thanked for facilitating testing in part of the study sample. The working group members of the Rehabilitation in MS Special Interest Group on Mobility are thanked for their consultation.

\section{References}

(1) Enright PL, Sherrill DL. Reference equations for the six-minute walk in healthy adults. Am J Respir Crit Care Med 1998 Nov;158:1384-1387.

(2) van Stel HF, Bogaard JM, Rijssenbeek-Nouwens LH, Colland VT. Multivariable assessment of the 6-min walking test in patients with chronic obstructive pulmonary disease. Am J Respir Crit Care Med 2001 Jun;163:1567-1571.

(3) Pohl PS, Perera S, Duncan PW, Maletsky R, Whitman R, Studenski S. Gains in distance walking in a 3-month follow-up poststroke: what changes? Neurorehabil Neural Repair 2004 Mar;18:30-36.

(4) Goldman MD, Marrie RA, Cohen JA. Evaluation of the six-minute walk in multiple sclerosis subjects and healthy controls. Mult Scler 2008 Apr;14:383-390.

(5) Paltamaa J, Sarasoja T, Leskinen E, Wikstrom J, Malkia E. Measuring deterioration in international classification of functioning domains of people with multiple sclerosis who are ambulatory. Phys Ther 2008 Feb;88:176-190.

(6) Gijbels D, Alders G, Van HE, et al. Predicting habitual walking performance in multiple sclerosis: relevance of capacity and self-report measures. Mult Scler 2010 May;16:618-626. 
(7) Butland RJ, Pang J, Gross ER, Woodcock AA, Geddes DM. Two-, six-, and 12minute walking tests in respiratory disease. $\mathrm{Br}$ Med J (Clin Res Ed) 1982 May 29;284:1607-1608.

(8) Kosak M, Smith T. Comparison of the 2-, 6-, and 12-minute walk tests in patients with stroke. J Rehabil Res Dev 2005 Jan;42:103-107.

(9) van den BM, Dawes H, Wade DT, et al. Treadmill training for individuals with multiple sclerosis: a pilot randomised trial. J Neurol Neurosurg Psychiatry 2006 Apr;77:531-533.

(10) Leung AS, Chan KK, Sykes K, Chan KS. Reliability, validity, and responsiveness of a 2-min walk test to assess exercise capacity of COPD patients. Chest 2006 Jul;130:119125. 
Figure 1. Mean walking distances of the $2 \mathrm{MWT}$ and $6 \mathrm{MWT}$ per 1-minute interval.

[Insert Figure 1 near here]

*Mean correlation coefficient $\mathrm{r}=0.99(p<0.01)$

Black vertical bars represent the standard error of the mean

Figure 2. The estimated 6MWT plotted against the actual 6MWT per subject.

[Insert Figure 2 near here] 


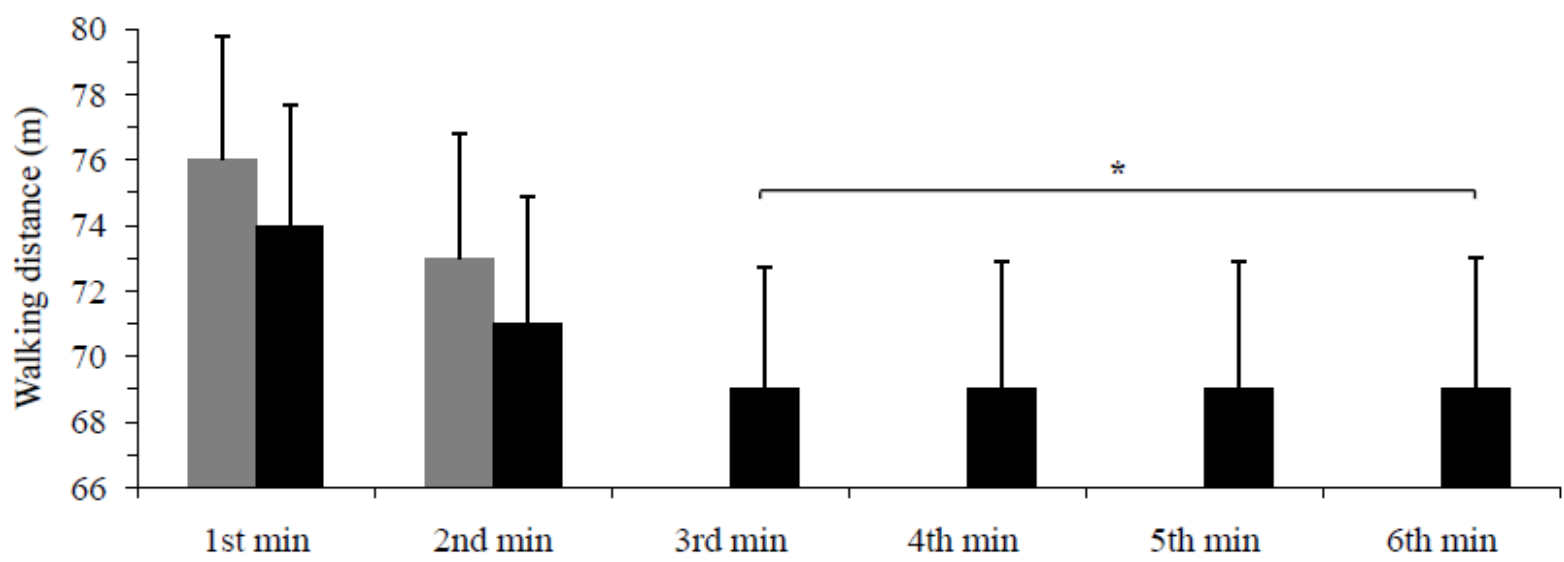

- Estimated 6MWT actual 6MWT

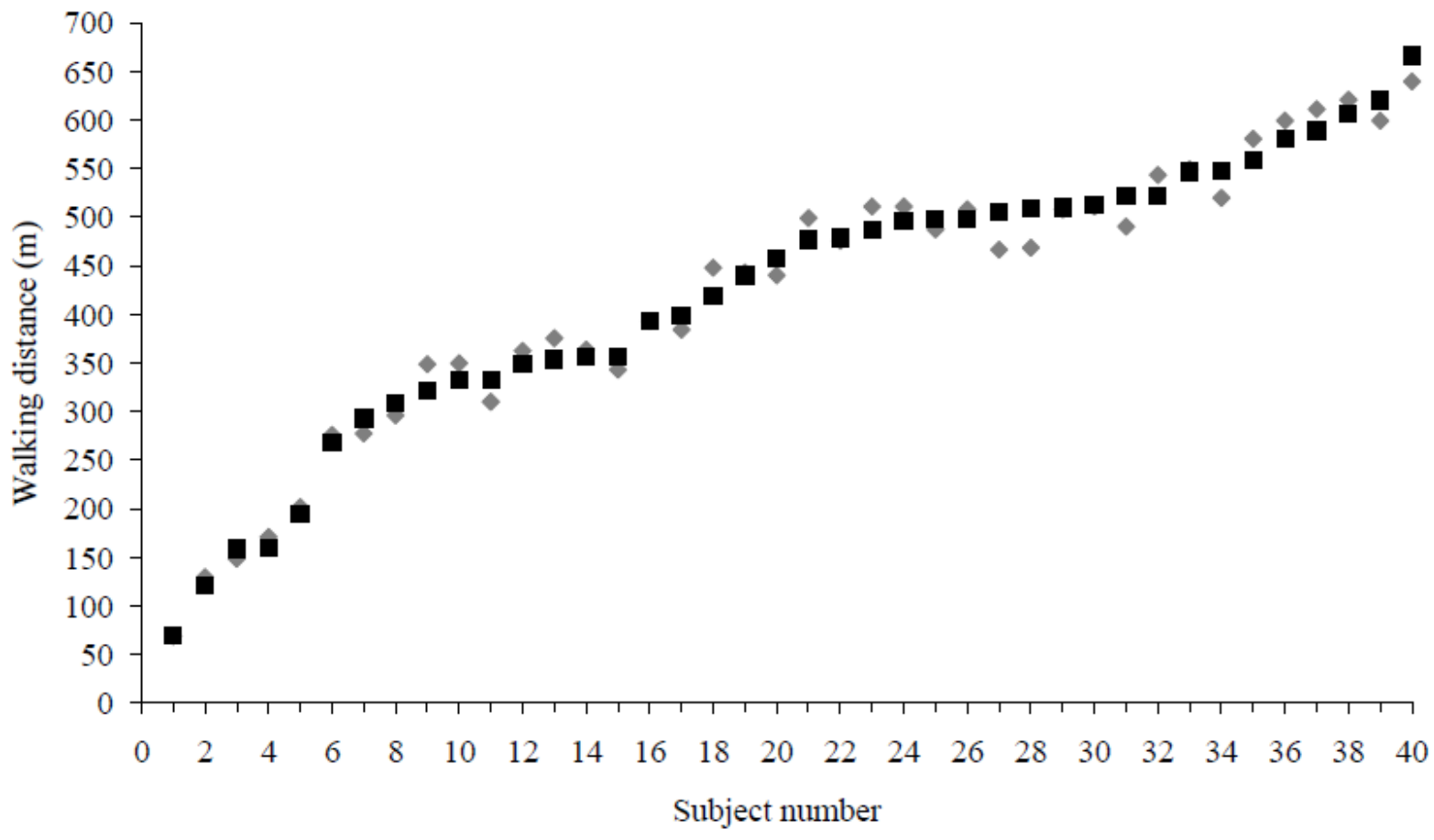

